\title{
Revisiter Reichenbach ? \\ Pour une approche sémantique systématique des temps verbaux de l'indicatif (en français)
}

\author{
Sophie Azzopardi ${ }^{1} \&$ Jacques Bres ${ }^{2}$ \\ ${ }^{1}$ Université Paris 7 Diderot, CLILLAC-ARP EA3967 \\ ${ }^{2}$ Université Paul Valéry Montpellier 3, Praxiling UMR 5267 CNRS \\ sophie.azzopardi@eila.univ-paris-diderot.fr, jacques.bres@univ-montp3.fr
}

\begin{abstract}
Résumé. Notre travail part du constat suivant : si de nombreux travaux se sont attachés à décrire le système verbal du français, notamment de l'indicatif, en proposant des modélisations fort abouties, aucune nous semblet-il n'a réussi à le faire pleinement à partir d'un nombre fini de paramètres. Nous tentons de le faire en prenant pour point de départ le système proposé par Reichenbach (1947) qui, en dépit des limites qu'il a montrées et des critiques pertinentes qu'il a suscitées, ne nous semble pas devoir être pour autant rejeté. Les trois points de son modèle $-\mathrm{S}$ : « speech point », $\mathrm{R}:$ « reference point »; et $\mathrm{E}:$ « event point »-sont redéfinis et les relations qu'il pose entre ces points sont complétées (section 2), afin de proposer (section 3 ) une modélisation qui permette de décrire, à partir des mêmes paramètres sémantiques, les formes simples, composées et périphrastiques de l'indicatif en français.
\end{abstract}

\begin{abstract}
This work takes issue with the description of the French verbal system. Many research works proposed models able to describe the French verbal system (mainly centered on the indicative), but none of them managed to make a complete description with a reduced number of parameters. With this work we try to do so starting from the system created by Reichebach (1947). This system, in spite of being criticized, doesn't have to be rejected. We give a new definition of the three points of the Reichenbach's model - S: «speech point», R: «reference point»; and $\mathrm{E}:$ : event point» - and complete the relations established between them (section 2). The aim is to propose a model based on the same semantic parameters and able to describe the simple tenses, the compound tenses and the periphrastic forms of the indicative French verbal system.
\end{abstract}




\section{Introduction}

Le système verbal du français, notamment de l'indicatif, a fait l'objet de nombreux travaux et plusieurs modélisations ont été élaborées au fil du temps, dont certaines extrêmement puissantes, notamment $i$. $a$. celles de Beauzée (1782), de Guillaume (1929), de Damourette et Pichon (1970[1911-1936]), de Wilmet (1970), de Martin (1971), ou plus récemment celle de Gosselin (1996, 2005). Malgré leur grand intérêt, aucune cependant ne nous apparaît pleinement satisfaisante, à savoir ne parvient à construire, à partir d'un petit nombre fini de paramètres, un système à même de rendre compte de l'unicité de la valeur en langue de chaque forme verbale, de sa différence par rapport aux formes voisines, comme de la pluralité de ses effets de sens en discours. Sans parler de la fréquente pierre d'achoppement que constitue l'intégration dans la description des périphrases verbales, notamment celles issues de la grammaticalisation en auxiliaires des verbes de déplacement aller et venir (Guillaume va partir / vient de partir en vacances) qui entrent en concurrence avec telle ou telle forme simple ou composée.

Notre présent propos est de reprendre le rocher sisyphéen du système des temps de l'indicatif, et de tenter de le faire rouler en prenant pour point de départ le système proposé par Reichenbach (1947). Nous n'ignorons pas que ledit système, après avoir fait naître beaucoup d'espoir, s'est avéré à la fois trop puissant théoriquement et défaillant concrètement : Vet 1980, 2007, Comrie 1983, Vikner 1985, Vetters 1996 et 2002, Saussure $1998 i$. a. en ont, de différentes manières, montré les limites. Ces critiques, pour pertinentes qu'elles soient, n'entraînent pas inéluctablement son rejet. Nous entendons bien plutôt le reprendre pour le réinvestir à nouveaux frais : les paramètres qu'il met en œuvre, au-delà du fait qu'ils sont largement utilisés dans différentes approches (cf. p. ex., outre Gosselin 1996 et 2005, Saussure 2010), ce qui assure la lisibilité de notre entreprise, nous paraissent une base solide sur laquelle développer une description des temps de l'indicatif qui ait quelque robustesse.

Après avoir présenté la modélisation de Reichenbach (1947) ainsi que le retravail que nous en faisons (section 2), nous décrirons le système que nous proposons des temps de l'indicatif (section 3$)^{\mathrm{i}}$.

\section{Reichenbach (1947)... augmenté}

\subsection{Reichenbach (1947) : les trois paramètres $S, E, R$, et les relations d'antériorité et de coïncidence}

Reichenbach (1947) part du constat suivant : pour placer un événement dans le temps, il est nécessaire d'avoir un point de repère à partir duquel il sera situé. De ce fait, le premier paramètre nécessaire à l'expression linguistique de l'événement est celui de l'acte d'énonciation. Ce que Reichenbach (1947 : 287-288) prend en compte, ce n'est pas l'acte d'énonciation en tant qu'événement, mais le moment $t_{0}$ où a lieu cette énonciation, appelé «point of speech » et noté S : 
The tenses determine time with reference to the time point of the act of speech, i.e., of the token uttered. [...] Let us call the time point of the token the point of speech.

La relation entre l'événement, plus précisément entre le "point of the event », noté E, et le point of speech S devrait permettre de le localiser sur la ligne du temps. Cependant, Reichenbach constate que de fait l'événement n'est pas situé directement par rapport au moment de l'énonciation, mais via la médiation d'un point de repère distinct, le «point of reference », noté $\mathrm{R}$ :

\begin{abstract}
From a sentence like 'Peter had gone' we see that the time order expressed in the tense does not concern one event, but two events, whose positions are determined with respect to the point of speech. We shall call these time points the point of the event and the point of reference. In the example the point of the event is the time when Peter went; the point of reference is a time between this point and the point of speech. (1947: 288)
\end{abstract}

Soient donc les trois points $E$ (event point), $S$ (speech point) et $R$ (reference point), qui permettent de formuler deux types de relations à partir de $\mathrm{R}$ : E et $\mathrm{R}$ d'une part (relation aspectuelle : l'aspect grammatical correspond à la façon dont est représenté $\mathrm{E}$ à partir du point $\mathrm{R}$, cf. infra 2.2.5. ), et $\mathrm{R}$ et $\mathrm{S}$ d'autre part (relation temporelle). Ces relations peuvent être d'antériorité $(-)$, ou de coïncidence (,). Dans ce cadre, le futur et le passé composé français p. ex. peuvent être décrits de la sorte :

(1) Guillaume partira en vacances : S - R, E

( $\mathrm{S}$ est antérieur à $\mathrm{R}$, qui coïncide avec $\mathrm{E}$ )

(2) Guillaume est parti en vacances : E - R, S

(E est antérieur à $\mathrm{R}$, qui coïncide avec $\mathrm{S}$ )

Reichenbach ajoute que si la représentation de l'événement lui-même (E) par un point semble convenir pour décrire certains temps verbaux comme le présent ou le prétérit, ce n'est pas le cas des formes qui donnent à voir l'événement dans sa durée, à savoir les formes progressives comme le present extended (I am seeing John) ou le present perfect extended (I have been seeing John) :

In some tenses, an additional indication is given concerning the time extension of the event. (op. cit.: 290)

Dans ce cas, Reichenbach propose de représenter l'événement non par un point, mais par ce que nous appellerons un segment ${ }^{\mathrm{ii}}$. Cette distinction point /segment lui permet également (1947: 291) de représenter la différence aspectuelle entre le passé simple et l'imparfait en français :
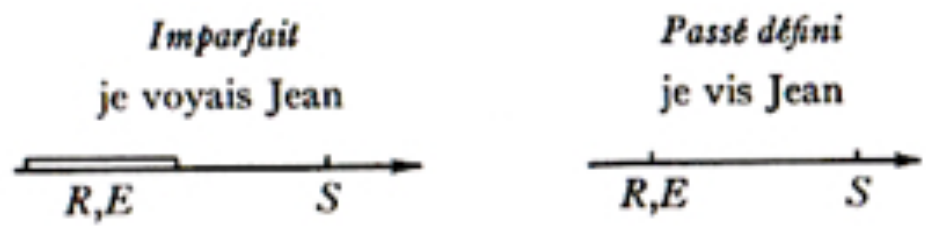

\title{
Schéma 1
}

Le système reichenbachien se signale par sa grande économie : trois points : $\mathrm{E}$, $\mathrm{R}$ et $\mathrm{S}$ (même si le premier est parfois non un point mais un « segment»), et deux relations : l'antériorité et la coïncidence. Ce qui a fait sa fortune. Mais cette économie de moyens permet-elle vraiment de décrire la temporalité verbale du français? Telle quelle, non. Prenons seulement le cas de l'imparfait : le schéma nous semble 
bien approximatif puisqu'il laisse entendre que $\mathrm{R}$, en relation de coïncidence avec $\mathrm{E}$, est également un segment temporel, alors que, en accord avec la grande majorité des approches, l'imparfait de « je voyais Jean » sera analysé comme représentant l'événement dans son cours à partir d'un point de référence. Soit donc une relation d'inclusion de $\mathrm{R}$ dans $\mathrm{E}$, qui n'est pas analysable à partir des seules notions d'antériorité et de coïncidence.

\subsection{Reichenbach retravaillé}

Notre faisons travailler le système de Reichenbach par des ajouts et des rectifications, qui affectent ses différents paramètres.

\subsubsection{Notions d' " event $E$ », de procès, de phases}

Lorsque Reichenbach parle d'event, il est bien difficile de savoir s'il parle de l'événement du monde auquel il est fait référence, ou de l'élément linguistique qui le signifie. Afin d'éviter pareille ambiguité, nous parlerons, dans la mesure où nous traitons non de l'événement du monde mais de sa représentation en langue, de procès (que cependant nous continuerons à désigner par $\mathrm{E}$, afin de maintenir le lien avec le système de Reichenbach).

Un procès peut être saisi selon trois phases (i.a. Dik 1989, Tournadre 2004, Gosselin 2011) : préprocessuelle, processuelle, et post-processuelle. Soit :

- L'event de Reichenbach correspond à la phase processuelle. Nous ajoutons deux éléments : correspondant à la phase pré-processuelle, $E^{\text {prep }}$; et correspondant à la phase post-processuelle, E ${ }^{\text {postp }}$. Soit

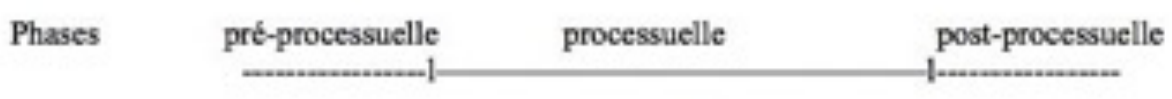

\section{Figure1}

donc pour l'analyse du procès, trois unités : $E^{\text {prep }}, E$, et $E^{\text {postp }}$.

- La phase processuelle est directement accessible par le seul verbe conjugué et s'effectue (principalement) au moyen de formes ${ }^{\mathrm{iii}}$ simples synthétiques (ex : présent, futur, imparfait...) (cf. cependant note 4 , et section 3.3.). Les phases pré-processuelle et post-processuelle, en revanche, ne peuvent être saisies directement et le sont nécessairement par le biais de formes analytiques qui allient un auxiliaire - pour une fois le terme de la grammaire est approprié - conjugué qui fonctionne comme une forme simple, et une forme quasi-nominale du verbe (participe, infinitif) ${ }^{\mathrm{iv}}$. On reconduira les termes de formes composées pour les temps construits à partir des auxiliaires avoir et être (ex : plus-que-parfait, futur antérieur...) et de formes périphrastiques pour les temps construits à partir d'auxiliaires tels que aller et venir $(\mathrm{de})^{\mathrm{v}} \mathrm{vi}$. Soit le tableau suivant :

\begin{tabular}{|c|c|c|c|}
\hline & Phase pré-processuelle & Phase processuelle & Phase post-processuelle \\
\hline $\begin{array}{c}\text { Formes simples } \\
\text { V. conjugué }\end{array}$ & $\varnothing$ & $\begin{array}{c}\text { ex : présent } \\
\text { Guillaume part en } \\
\text { vacances }\end{array}$ & $\varnothing$ \\
\hline $\begin{array}{c}\text { Formes composées } \\
\text { auxiliaire conjugué } \\
+ \text { V. participe passé }\end{array}$ & $\varnothing$ & $\varnothing$ & $\begin{array}{c}\text { ex : plus-que-parfait } \\
\text { Guillaume était parti en } \\
\text { vacances }\end{array}$ \\
\hline
\end{tabular}




\begin{tabular}{|c|c|c|c|}
\hline $\begin{array}{l}\text { Formes périphrastiques } \\
\text { auxiliaire conjugué } \\
+\mathrm{V} \text {. INF ou gérondif }\end{array}$ & $\begin{array}{c}\text { ex : aller }+\mathrm{INF} \\
\text { Guillaume va partir en } \\
\text { vacances }\end{array}$ & $\begin{array}{c}\text { ex : être en train de }+ \\
\text { INF } \\
\text { Guillaume est en train de } \\
\text { partir en vacances }\end{array}$ & $\begin{array}{c}\text { ex : venir de }+\mathrm{INF} \\
\text { Guillaume vient de partir en } \\
\text { vacances }\end{array}$ \\
\hline
\end{tabular}

\subsubsection{Notions de point et d'intervalle}

Reichenbach parle pour les trois paramètres $\mathrm{S}, \mathrm{R}$ et $\mathrm{E}$, de points, même si le point $\mathrm{E}$ tend à devenir un « segment » pour les formes progressives en anglais ou l'imparfait en français, comme nous l'avons vu. Ce glissement du point à une unité dotée d'une épaisseur nous semble être le signe d'une difficulté.

C'est que considérer les trois paramètres $\mathrm{E}, \mathrm{R}$, et $\mathrm{S}$ comme des points ne permet pas de saisir pleinement la dimension aspectuelle du procès, dans la mesure où on ne saurait représenter un point sous différents aspects. Pour remédier à ce problème, Klein (1994), Gosselin (1996) et Bres (2015) ont proposé, dans des cadres théoriques différents, de concevoir $\mathrm{E}, \mathrm{R}$ et $\mathrm{S}$ non comme des points mais comme des intervalles, avec borne initiale et borne terminale. Notre réflexion actuelle nous conduit à conserver le statut de points pour $\mathrm{R}$ et $\mathrm{S}$, et à analyser $\mathrm{E}$ comme un intervalle. Cette différence de traitement se justifie de ce que :

- S, en tant que «point of speech », correspond à l'acte d'énonciation non dans sa durée mais dans sa survenue au temps ;

$-\mathrm{R}$, en tant que point de référence, est une pure position à partir de laquelle est saisi le procès $\mathrm{E}$, de différentes façons selon la position de $\mathrm{R}$ par rapport à $\mathrm{E}$ (et non, comme chez Gosselin, un intervalle correspondant à ce qui est « montré » du procès) ;

- E, au contraire, parce qu'en tant que procès il réfère à un événement du monde, implique du temps (Guillaume 1969 (1933) : 47), un temps que Comrie (1976:5) nomme "internal time ». Nous le représentons par un intervalle avec borne initiale (notée ${ }_{i}$ ) et borne terminale (notée $t_{t}$ ), soit $\left(\mathrm{E}_{\mathrm{i}}-\mathrm{E}_{\mathrm{t}}\right.$ ). Nous verrons que cette représentation permet de bien distinguer l'imparfait du passé simple. Cet intervalle peut être réduit à un point lorsque le temps interne au procès n'est pas pris en compte dans la représentation linguistique qui est donnée de l'événement (ce qui est le cas du passé simple p. ex.).

D'autre part, nous avons distingué supra, outre la phase processuelle, les phases pré- et post-

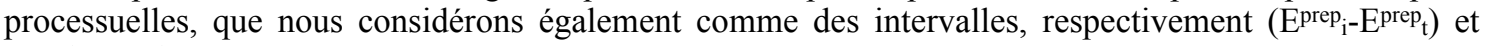
$\left(E^{\text {postp }}{ }_{i} E^{\text {postp }}{ }_{t}\right)$. Ces phases sont prises en charge par les formes composées et les formes périphrastiques. Morphologiquement, ces formes comportent deux éléments : un auxiliaire conjugué + un verbe au participe passé ou à l'infinitif. À la différence des formes simples pour lesquelles un seul intervalle, l'intervalle processuel $\left(E_{i}-E_{t}\right)$, est concerné, les formes composées et périphrastiques, du fait de la présence de deux morphèmes (un auxiliaire + un participe passé, un infinitif), mettent en jeu deux intervalles de procès : comme dans les formes simples, l'intervalle dénoté par le verbe à la forme quasinominale $\left(E_{\mathrm{i}}-\mathrm{E}_{\mathrm{t}}\right)$; et l'intervalle dénoté par l'auxiliaire conjugué $\left(\varepsilon_{\mathrm{i}-} \varepsilon_{\mathrm{t}}\right)$. C'est la conjonction, dans la forme composée ou périphrastique, de $\left(\varepsilon_{\mathrm{i}}-\varepsilon_{\mathrm{t}}\right)+\left(\mathrm{E}_{\mathrm{i}}-\mathrm{E}_{\mathrm{t}}\right)$ qui fait que Guillaume va partir en vacances donne à voir le procès partir dans sa phase pré-processuelle ; et que Guillaume est parti en vacances donne à voir le procès partir dans sa phase post-processuelle (infra 3.2.2.).

Ajoutons que les trois phases étant connexes, la borne terminale $\varepsilon_{\mathrm{t}}$ de l'intervalle de l'auxiliaire servant à la construction de la phase pré-processuelle correspond à la borne initiale de la phase processuelle $\mathrm{E}_{\mathrm{i}}$; et la borne initiale $\varepsilon_{i}$ de l'intervalle de l'auxiliaire servant à la construction de la phase post-processuelle correspond à la borne terminale de la phase processuelle $\mathrm{E}_{\mathrm{t}}$.

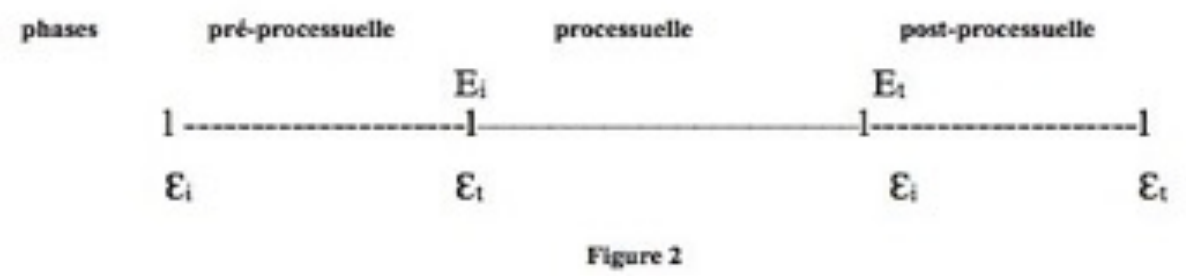




\subsubsection{Point de référence $R$ et point de référence $\rho$}

Comme l'intervalle $E_{i}-E_{t}$ du procès est représenté à partir d'un point de référence $R$, l'intervalle $\varepsilon_{i}-\varepsilon_{t}$ de l'auxiliaire (être, avoir, aller, venir) qui permet de saisir le procès dans ses phases pré-processuelle ( $\mathrm{E}^{\text {prep }_{i}-}$ $E^{\text {prep }}{ }_{t}$ ) et post-processuelle ( $\left.E^{\text {postp }}{ }_{i}-E^{\text {postp }}{ }_{t}\right)$ dispose d'un point de référence que nous représentons par la lettre grecque $\rho$, dont on verra l'importance et la nécessité infra dans la description des temps composés et périphrastiques.

\subsubsection{Point d'énonciation $S$ et point d'énonciation S'}

La complexification du système de Reichenbach que nous avons présentée pour les paramètres $\mathrm{E}$ et $\mathrm{R}$ affecte également le paramètre S. Pour le philosophe, il n'y a qu'un seul "point of speech ». La réalité des temps verbaux, plus précisément du conditionnelvii, est plus complexe. Le conditionnel, au moins dans son emploi temporel - Marie a dit que Guillaume partirait en vacances - pose en ultériorité un procès non pas à partir de l'énonciation du locuteur-énonciateur mais à partir d'une énonciation rapportée, celle de Marie dans l'exemple proposéviiii. Cette énonciation rapportée dispose d'un point of speech propre, que nous nommons S', et qui, dans le cas du conditionnel, est antérieur à S (soit donc S'$\mathrm{S})$. Ce dédoublement du point of speech, nécessaire pour décrire le conditionnel en langue, s'avère également utile pour décrire certains emplois dialogiques ${ }^{\mathrm{ix}}$ de l'imparfait ou du futur en discours.

\subsubsection{Relations d'inclusion, de neutralité et de proximité immédiate}

Pour rendre compte des relations (aspectuelles) entre $\mathrm{R}$ et $\mathrm{E}$ en français, il faut ajouter, aux relations d'antériorité et de coïncidence ${ }^{\mathrm{x}}$ de Reichenbach, les relations d'inclusion, de neutralité et d'antériorité proche :

- la relation d'inclusion ( $\subset$ ) permet de distinguer l'imparfait (représentation cursive) du passé simple (représentation globale), (comme d'ailleurs le plus-que-parfait du passé antérieur) :

(3) Guillaume partait en vacances $: R \subset E_{i-} E_{t} ; R-S$

(le point de référence $\mathrm{R}$ est inclus dans l'intervalle du procès $\mathrm{E}$ et est antérieur au point d'énonciation $S$ )

(4) Guillaume partit en vacances : R $=E$; R $-S$

(le point de référence $\mathrm{R}$ coïncide avec l'intervalle du procès $\mathrm{E}$ réduit à un point et est antérieur au point d'énonciation $\mathrm{S}$ )

- la relation de neutralité $(\subseteq)$ permet de décrire, à côté des temps où $\mathrm{R}$ est inclus dans $\mathrm{E}$ (imparfait : $\mathrm{R} \subset$ $\mathrm{E}$ ) ou coïncide avec $\mathrm{E}$ (passé simple : $\mathrm{R}=\mathrm{E}$ ), des temps comme le présent ou le futur où la relation de $\mathrm{R}$ avec $\mathrm{E}$ est sous-déterminée ( $\subseteq \mathrm{E}$ ), à savoir qui ne marquent pas si $\mathrm{R}$ est inclus dans ou coïncide avec $\mathrm{E}$

(5) Guillaume partira en vacances : $\mathrm{S}-\mathrm{R} \subseteq \mathrm{E}_{\mathrm{i}}-\mathrm{E}_{\mathrm{t}}$

(le point d'énonciation $\mathrm{S}$ est antérieur au point de référence qui, suivant le contexte, sera inclus dans l'événement ou coïncidera avec lui)

- la relation d'antériorité entre $\mathrm{E}$ et $\mathrm{R}$, contrairement à ce qu'induit le modèle de Reichenbach, peut être non seulement qualitative, mais également quantitative (Vetters 2002 : 115-118), et signifier des degrés de distance (Comrie 1985 : 83-101), notamment pour le français, une petite distance temporelle. On représentera la relation d'antériorité proche entre $\mathrm{E}$ et $\mathrm{R}$ par le signe $(<)$, ce qui permet notamment de distinguer d'une part futur (6) et ultériorité proche (6a), et d'autre part passé composé (7) et antériorité proche (7a). Soit, dans une représentation provisoire :

(6) Guillaume partira en vacances : $\mathrm{S}-\mathrm{R} \subseteq \mathrm{E}_{\mathrm{i}}-\mathrm{E}_{\mathrm{t}}$ 
( $\mathrm{S}$ est antérieur à $\mathrm{R}$, qui est dans une relation sous-déterminée avec $\mathrm{E}$ )

(6a) Guillaume va partir en vacances: $\mathrm{S}=\mathrm{R}<\mathrm{Ex}^{\mathrm{xi}}$

( $\mathrm{S}$ coïncide avec $\mathrm{R}$, qui est proximalement antérieur à $\mathrm{E}$ )

(7) Guillaume est parti en vacances : $\mathrm{E}_{\mathrm{i}}-\mathrm{E}_{\mathrm{t}}-\mathrm{R}=\mathrm{S}$

(E est antérieur à $\mathrm{R}$, qui coïncide avec $\mathrm{S}$ )

(7a) Guillaume vient de partir en vacances : $\mathrm{E}<\mathrm{R}=\mathrm{S}$

(E est proximalement antérieur à $\mathrm{R}$, qui coïncide avec $\mathrm{S}$ )

- Nous disposons, dans le système de Reichenbach, de la relation de coïncidence : elle prend en charge la relation temporelle entre les points $\mathrm{S}$ et $\mathrm{R}$ p. ex. dans le présent, ou la relation aspectuelle entre $\mathrm{R}$ et $\mathrm{E}$ (intervalle réduit à un point) comme dans le passé simple. Mais la coïncidence peut se réaliser également entre $R$ et un point de $E_{i}-E_{t}$, celui de la borne terminale de l'intervalle du procès $E_{t}$, dans le cas du participe passé, soit $\mathrm{R}=\mathrm{E}_{\mathrm{t}}$.

On dispose donc de 5 relations : antériorité $(-)$, coïncidence $(=)$, inclusion $(\subset)$, neutralité $(\subseteq)$, antériorité proche $(<)$, qui, nous le verrons infra, permettent de décrire la totalité des relations entre $\mathrm{R}$ et $\mathrm{E}$ d'une part, et entre $\mathrm{R}$ et $\mathrm{S}$ d'autre part.

Résumons notre retravail du système de Reichenbach :

- nous considérons l'élément $\mathrm{E}$, non comme un point (à la différence de $\mathrm{R}$ et de $\mathrm{S}$ ) mais comme un intervalle borné $\left(\mathrm{E}_{\mathrm{i}}-\mathrm{E}_{\mathrm{t}}\right)$ (pouvant éventuellement se réduire à un point, comme dans le cas du passé simple);

- l'élément E étant un procès, il peut être représenté dans sa phase processuelle mais également dans sa phase pré-processuelle $E^{\text {prep }}$ ou dans sa phase post-processuelle Epostp. À cela servent les auxiliaires, qui disposent d'un intervalle noté $\left(\varepsilon_{\mathrm{i}}-\varepsilon_{\mathrm{t}}\right)$;

- tout comme la phase processuelle $E_{i}-E_{t} d u$ verbe dispose d'un point de référence $R$, la phase processuelle $\varepsilon_{\mathrm{i}}-\varepsilon_{\mathrm{t}}$ des auxiliaires dispose d'un point de référence $\rho$;

- dans les cas de dialogisme, le point S doit être doublé d'un point of speech S';

- aux relations d'antériorité $(-)$ et de coïncidence $(=)$, nous ajoutons les relations d'inclusion $(\subset)$, de neutralité $(\subseteq)$ et d'antériorité proche $(<)$.

Cette complexification pourra paraître fort éloignée du système de Reichenbach, voire n'avoir plus rien à voir avec lui. Nous ne le pensons pas : soulignons que nous en gardons les trois piliers E, R, S ainsi que leur mise en relation deux à deux : $\mathrm{E} / \mathrm{R}$ (relation aspectuelle), et $\mathrm{R} / \mathrm{S}$ (relation temporelle).

\section{Description du système des temps verbaux de l'indicatif}

Morphologiquement, l'indicatif comporte des formes simples, des formes composées (être ou avoir + pp.), des formes périphrastiques (aller, venir (de), être en train de etc. + inf.). Nous l'avons vu : les formes simples prennent exclusivement en charge la représentation de la phase processuelle, les formes composées et périphrastiques prennent principalement en charge la représentation des phases préprocessuelle et post-processuelle. 


\subsection{Formes simples}

Les temps simples (présent, imparfait, passé simple, futur simple, conditionnel présent) prennent en charge la représentation de la phase processuelle. La relation $\mathrm{E} / \mathrm{R}$ détermine la représentation aspectuelle ; la relation $\mathrm{S} / \mathrm{R}$, la localisation temporelle.

\begin{tabular}{|c|c|c|}
\hline Temps & Représentation aspectuelle & Localisation temporelle \\
\hline présent & sous-déterminée $\left(\mathrm{R} \subseteq \mathrm{E}_{\mathrm{i}}-\mathrm{E}_{\mathrm{t}}\right)$ & présent $(\mathrm{R}=\mathrm{S})$ \\
\hline futur & sous-déterminée $\left(\mathrm{R} \subseteq \mathrm{E}_{\mathrm{i}}-\mathrm{E}_{\mathrm{t}}\right)$ & ultérieur du présent $(\mathrm{S}-\mathrm{R})$ \\
\hline passé simple & globale $(\mathrm{R}=\mathrm{E})$ & passé $(\mathrm{R}-\mathrm{S})$ \\
\hline Imparfait & cursive $\left(\mathrm{R} \subset \mathrm{E}_{\left.\mathrm{i}-\mathrm{E}_{\mathrm{t}}\right)}\right.$ & passé $(\mathrm{R}-\mathrm{S})$ \\
\hline conditionnel & sous-déterminée $\left(\mathrm{R} \subseteq \mathrm{E}_{\mathrm{i}}-\mathrm{E}_{\mathrm{t}}\right)$ & ultérieur du passé $\left(\mathrm{S}^{\prime}-\mathrm{R} ; \mathrm{S}^{\prime}-\mathrm{S}\right)$ \\
\hline
\end{tabular}

\section{Tableau des formes simples}

Les formules données dans le tableau peuvent être explicitées ainsi :

Présent : Guillaume part en vacances : $\mathrm{R} \subseteq \mathrm{E}_{\mathrm{i}}-\mathrm{E}_{\mathrm{t}} ; \mathrm{R}=\mathrm{S}$

Le procès partir est saisi dans sa phase processuelle, globalement ou cursivement selon le co(n)texte (représentation aspectuelle sous-déterminée, $\mathrm{R} \subseteq \mathrm{E}_{\mathrm{i}}-\mathrm{E}_{\mathrm{t}}$ ); cette saisie est située dans l'époque présente (localisation temporelle dans laquelle le point de référence coïncide avec l'énonciation, $\mathrm{R}=\mathrm{S}$ ).

Futur : Guillaume partira en vacances : $\mathrm{R} \subseteq \mathrm{E}_{\mathrm{i}}-\mathrm{E}_{\mathrm{t}} ; \mathrm{S}-\mathrm{R}$

Le procès partir est saisi dans sa phase processuelle, globalement ou cursivement selon le co(n)texte (représentation aspectuelle sous-déterminée, $\mathrm{R} \subseteq \mathrm{E}_{\mathrm{i}}-\mathrm{E}_{\mathrm{t}}$ ) ; cette saisie est située dans l'époque future (localisation temporelle dans laquelle l'énonciation est antérieure au point de référence, $\mathrm{S}-\mathrm{R}$ ).

$$
\text { Passé simple : Guillaume partit en vacances : } \mathrm{R}=\mathrm{E} ; \mathrm{R}-\mathrm{S}
$$

Le procès partir est saisi dans sa phase processuelle dans sa globalité réduite à un point (représentation aspectuelle globale, $\mathrm{R}=\mathrm{E}$ ) ; cette saisie est située dans l'époque passée (localisation temporelle dans laquelle le point de référence est antérieur à l'énonciation, $\mathrm{R}-\mathrm{S}$ ).

$$
\text { Imparfait : Guillaume partait en vacances : } \mathrm{R} \subset \mathrm{E}_{\mathrm{i}}-\mathrm{E}_{\mathrm{t}} ; \mathrm{R}-\mathrm{S}
$$

Le procès partir est saisi dans son cours (représentation aspectuelle cursive, $\mathrm{R} \subset \mathrm{E}$ ) ; cette saisie est située dans l'époque passée (localisation temporelle dans laquelle le point de référence est antérieur à l'énonciation, $\mathrm{R}-\mathrm{S}$ ).

$$
\text { Conditionnel : (Marie avait dit que) Guillaume partirait en vacances : } R \subseteq E_{i}-E_{t} ; S^{\prime}-R ; S^{\prime}-S
$$

Le procès partir est saisi dans sa phase processuelle globalement ou cursivement selon le co(n)texte (représentation aspectuelle sous-déterminée, $\mathrm{R} \subseteq \mathrm{E}_{\mathrm{i}}-\mathrm{E}_{\mathrm{t}}$ ) ; cette saisie est située dans l'ultériorité d'une énonciation secondaire S', elle-même située dans l'antériorité de l'énonciation principale (localisation temporelle dans laquelle l'énonciation secondaire $S$ ' est antérieure au point de référence, $S$ ' - R; et est également antérieure à l'énonciation principale, $\mathrm{S}$ ' $-\mathrm{S}$ ).

Notons que, dans le cas du conditionnel, le point de référence étant positionné par rapport à une énonciation $S$ ' antérieure à $S$, la relation entre $R$ et $S$ n'est pas contrainte. C'est ce qui permet de rendre 
compte du fait que le procès partir puisse être situé dans l'époque passée $(R-S)$, présente $(S=R)$ ou future $(\mathrm{S}-\mathrm{R})$ :

(8) Marie avait dit que Guillaume partirait en vacances hier / maintenant / demain

Nous allons à présent décrire le fonctionnement des formes composées et périphrastiques.

\subsection{Formes composées et périphrastiques}

Les formes composées (être/avoir + pp.) et les formes périphrastiques (ex : aller + inf., venir de + inf.) permettent la représentation des phases pré-processuelle et post-processuelle. Morphologiquement, ces formes comportent deux éléments : un auxiliaire conjugué + un verbe au pp. ou à l'inf. À la différence des formes simples pour lesquelles un seul intervalle, l'intervalle processuel $\left(E_{\mathrm{i}}-\mathrm{E}_{\mathrm{t}}\right)$, est concerné, les formes composées et périphrastiques, du fait de la présence de deux morphèmes (un auxiliaire + un $\mathrm{V}$ ), mettent en jeu deux intervalles : comme dans les temps simples, l'intervalle processuel $\mathrm{E}_{\mathrm{i}} \mathrm{E}_{\mathrm{t}}$ dénoté par le verbe à la forme quasi-nominale ; et l'intervalle processuel $\varepsilon_{\mathrm{i}}-\varepsilon_{\mathrm{t}}$ dénoté par l'auxiliaire. C'est la conjonction de $\left(\varepsilon_{\mathrm{i}}-\varepsilon_{\mathrm{t}}\right)+\left(\mathrm{E}_{\mathrm{i}}-\mathrm{E}_{\mathrm{t}}\right)$ qui fait que Guillaume va partir en vacances donne à voir le procès partir dans sa phase pré-processuelle ; et que Guillaume est parti en vacances donne à voir le procès partir dans sa phase post-processuelle.

\subsubsection{Formes composées}

Dans les formes composées,

- le pp. du verbe donne seulement une instruction aspectuelle : celle de saisir l'intervalle $E_{i}-E_{t}$ sur sa borne terminale : le point de référence coïncide avec ladite borne $: \mathrm{R}=\mathrm{E}_{\mathrm{t}}$. Le participe passé, en tant que forme nominale, est atemporel (Guillaume 1929) : il ne donne donc pas d'instruction temporelle ;

- l'auxiliaire, comme toute forme conjuguée dans sa phase processuelle, donne une instruction aspectuelle (relation entre $\rho$ et $\varepsilon_{\mathrm{i}}-\varepsilon_{\mathrm{t}}$ ) et une instruction temporelle (relation entre $\rho$ et $\mathrm{S}$ ).

$\rightarrow$ aux + V. au pp. : en tant qu'auxiliaires, avoir/être n'ont pas un sens plein et le procès qu'ils saisissent n'a pas de référent dans la réalité extra-linguistique. En interaction avec le fait que la phase processuelle $\mathrm{du}$ V. est saisie par le pp. sur sa borne terminale $\left(R=E_{t}\right)$, l'intervalle $\varepsilon_{\mathrm{i}}-\varepsilon_{\mathrm{t}}$ de l'auxiliaire coïncide avec la phase post-processuelle $E^{\text {postp. }}$ Le procès est actualisé dans sa phase post-processuelle, localisée temporellement via la médiation du point de référence $\rho$ de l'auxiliaire.

\begin{tabular}{|c|c|c|}
\hline Temps & Représentation aspectuelle & Localisation temporelle \\
\hline V. au participe passé & $\mathrm{R}=\mathrm{E}_{\mathrm{t}}$ & $\varnothing$ \\
\hline aux. au présent & sous-déterminée $\left(\rho \subseteq \varepsilon_{\mathrm{i}-} \varepsilon_{\mathrm{t}}\right)$ & présent $(\rho=S)$ \\
\hline$\rightarrow$ passé composé & 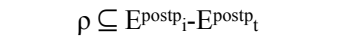 & présent $(\rho=S)$ \\
\hline V. au participe passé & $\mathrm{R}=\mathrm{E}_{\mathrm{t}}$ & $\varnothing$ \\
\hline aux. au futur & sous-déterminée $\left(\rho \subseteq \varepsilon_{\mathrm{i}}-\varepsilon_{\mathrm{t}}\right)$ & ultérieur du présent $(S-\rho)$ \\
\hline$\rightarrow$ futur antérieur & $\rho \subseteq \mathrm{E}^{\text {postp }} \mathrm{i}_{\mathrm{i}}-\mathrm{E}^{\text {postp }} \mathrm{t}$ & ultérieur du présent $(S-\rho)$ \\
\hline V. au participe passé & $\mathrm{R}=\mathrm{E}_{\mathrm{t}}$ & $\varnothing$ \\
\hline aux. à l'imparfait & cursive $\left(\rho \subset \varepsilon_{\mathrm{i}}-\varepsilon_{\mathrm{t}}\right)$ & passé $(\rho-S)$ \\
\hline$\rightarrow$ plus-que-parfait & cursive $\left(\rho \subset E^{\text {postp }_{i}-E^{\text {postp }}}{ }_{t}\right)$ & passé $(\rho-S)$ \\
\hline
\end{tabular}




\begin{tabular}{|c|c|c|}
\hline V. au participe passé & $\mathrm{R}=\mathrm{E}_{\mathrm{t}}$ & $\varnothing$ \\
\hline Aux. au passé simple & globale $(\rho=\varepsilon)$ & passé $(\rho-S)$ \\
\hline$\rightarrow$ passé antérieur & globale $\left(\rho=E^{\text {postp }}\right)$ & passé $(\rho-S)$ \\
\hline V. au participe passé & $\mathrm{R}=\mathrm{E}_{\mathrm{t}}$ & $\varnothing$ \\
\hline aux. au conditionnel & sous-déterminée ( $\rho \subseteq \varepsilon_{\mathrm{i}}-\varepsilon_{\mathrm{t}}$ ) & ultérieur du passé $\left(S^{\prime}-S ; S^{\prime}-\rho\right)$ \\
\hline$\rightarrow$ conditionnel passé & 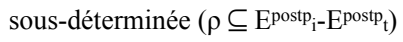 & ultérieur du passé $\left(S^{\prime}-\rho ; S^{\prime}-S\right)$ \\
\hline
\end{tabular}

Tableau des formes composées

Les formules données dans le tableau peuvent être explicitées ainsi :

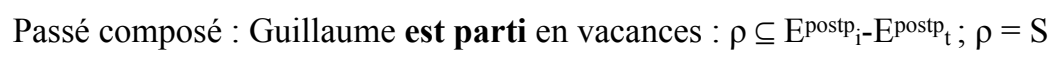

Le passé composé, combinaison d'un auxiliaire au présent et du V. au participe passé, saisit le procès dans sa phase post-processuelle, globalement ou cursivement selon le co(n)texte (représentation aspectuelle sous-déterminée, $\rho \subseteq \mathrm{E}^{\text {postp}} \mathrm{i}_{\mathrm{i}} \mathrm{E}^{\text {post }} \mathrm{t}$ ) ; cette saisie est située dans l'époque présente (localisation temporelle dans laquelle le point de référence $\rho$ coïncide avec l'énonciation principale $S, \rho=S$ ).

Futur antérieur : Guillaume sera parti en vacances : $\rho \subseteq E^{\text {postp }_{i}-E^{\text {post }}} p_{t} ; S-\rho$

Le futur antérieur, combinaison d'un auxiliaire au futur et du V. au participe passé, saisit le procès dans sa phase post-processuelle, globalement ou cursivement selon le co(n)texte (représentation aspectuelle sous-

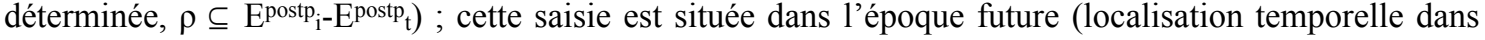
laquelle l'énonciation principale est antérieure au point de référence, $S-\rho$ ).

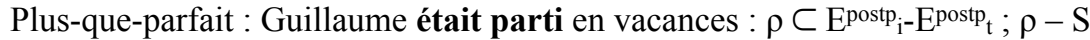

Le plus-que-parfait, combinaison d'un auxiliaire à l'imparfait et du V. au participe passé, saisit le procès dans sa phase post-processuelle cursivement (représentation aspectuelle cursive, $\rho \subset E^{\text {post }_{i}-E^{\text {post }}}{ }_{t}$ ); cette saisie est située dans l'époque passée (localisation temporelle dans laquelle le point de référence est antérieur à l'énonciation principale, $\rho-\mathrm{S}$ ).

Passé antérieur : (quand) Guillaume fut parti en vacances : $\rho=E^{\text {postp }} ; \rho-S$

Le passé antérieur, combinaison d'un auxiliaire au passé simple et du V. au participe passé, saisit le procès dans sa phase post-processuelle dans sa globalité réduite à un point (représentation aspectuelle globale, $\rho=\mathrm{E}^{\text {postp) }}$; cette saisie est située dans l'époque passée (localisation temporelle dans laquelle le point de référence est antérieur à l'énonciation principale, $\rho-S)$.

Conditionnel passé : (Marie avait dit que) Guillaume serait parti en vacances :

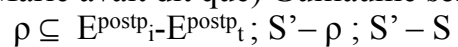

Le conditionnel passé, combinaison d'un auxiliaire au conditionnel présent et du V. au participe passé, saisit le procès dans sa phase post-processuelle, globalement ou cursivement selon le co(n)texte

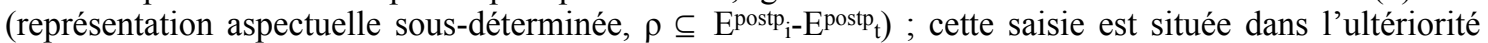
d'une énonciation secondaire $\left(S^{\prime}-\rho\right)$, elle-même située dans l'antériorité de l'énonciation principale ( $S^{\prime}-$ S). Notons que, comme dans le cas du conditionnel présent, le point de référence $\rho$ étant positionné par rapport à une énonciation $S$ ' antérieure à $S$, la relation entre $\rho$ et $S$ n'est pas contrainte. C'est ce qui permet de rendre compte du fait que la phase post-processuelle du procès partir puisse être située dans le passé $(\rho-S)$, le présent $(S=\rho)$ ou le futur $(S-\rho)$ :

(9) Marie avait dit que Guillaume serait parti en vacances hier / maintenant / demain

Nous allons à présent décrire le fonctionnement des formes périphrastiques. 


\subsubsection{Formes périphrastiques}

Dans les formes périphrastiques,

- l'infinitif du verbe donne seulement une instruction aspectuelle : il saisit le procès globalement ou cursivement selon le co(n)texte (représentation aspectuelle sous-déterminée, $\mathrm{R} \subseteq \mathrm{E}_{\mathrm{i}}-\mathrm{E}_{\mathrm{t}}$ ). L'infinitif, en tant que forme nominale, est atemporel (Guillaume 1929) : il ne donne pas d'instruction temporelle ;

- l'auxiliaire, comme toute forme conjuguée dans sa phase processuelle, donne une instruction aspectuelle et une instruction temporelle. Le sémantisme de l'auxiliaire indique la phase de E qui sera saisie par la forme périphrastique.

Comme nous l'avons indiqué supra, nous ne décrirons ici que le fonctionnement de aller + infinitif et de venir de + infinitif à titre d'exemple de formes périphrastiques prenant en charge respectivement les phases pré- et post-processuelles de $\mathrm{E}$.

\subsubsection{Aller + V. infinitif}

Aller, en tant que verbe, signifie le mouvement vers un point donné. Grammaticalisé en auxiliaire, il indique le mouvement vers la phase processuelle du procès dénoté et permet de le saisir dans sa phase pré-processuelle, à proximité de sa phase processuelle. Notons, sans l'expliquer pour l'heure, que aller, dans ce fonctionnement, est défectif : il ne peut se conjuguer qu'au présent et à l'imparfait.

\begin{tabular}{|c|c|c|c|}
\hline Temps & Représentation aspectuelle & Localisation temporelle \\
\hline \multirow{2}{*}{ aux. au présent } & V. à l'infinitif & $\mathrm{R} \subseteq \mathrm{E}_{\mathrm{i}}-\mathrm{E}_{\mathrm{t}}$ & $\varnothing$ \\
\cline { 2 - 4 } & $\rho \subseteq \varepsilon_{\mathrm{i}}-\varepsilon_{\mathrm{t}}$ & $\rho=\mathrm{S}$ \\
\hline$\rightarrow$ présent prospectif & $\rho<\mathrm{E}_{\mathrm{i}}$ & $\rho=\mathrm{S}$ \\
\hline & V. à l'infinitif & $\mathrm{R} \subseteq \mathrm{E}_{\mathrm{i}}-\mathrm{E}_{\mathrm{t}}$ & $\varnothing$ \\
\cline { 2 - 4 } & aux. à l'IMP & $\rho \subset \varepsilon_{\mathrm{i}}-\varepsilon_{\mathrm{t}}$ & $\rho-\mathrm{S}$ \\
\hline & imp. prospectif & $\rho<\mathrm{E}_{\mathrm{i}}$ & $\rho-\mathrm{S}$ \\
\hline
\end{tabular}

Les formules données dans le tableau peuvent être explicitées ainsi :

Présent prospectif : Guillaume va partir en vacances : $\rho<\mathrm{E}_{\mathrm{i}} ; \rho=\mathrm{S}$

Le présent prospectif, combinaison de l'auxiliaire aller au présent et du V. à l'infinitif, saisit le procès dans sa phase pré-processuelle, en un point de référence à proximité de la borne initiale de la phase processuelle du procès $\left(\rho<\mathrm{E}_{\mathrm{i}}\right)$, saisie située dans l'époque présente (localisation temporelle dans laquelle le point de référence $\rho$ coïncide avec l'énonciation principale $S, \rho=S$ ).

Imparfait prospectif : Guillaume allait partir en vacances : $\rho<\mathrm{E}_{\mathrm{i}} ; \rho-\mathrm{S}$

L'imparfait prospectif, combinaison de l'auxiliaire aller à l'imparfait et du V. à l'infinitif, saisit le procès dans sa phase pré-processuelle, en un point de référence à proximité de la borne initiale de la phase processuelle du procès $\left(\rho<\mathrm{E}_{\mathrm{i}}\right)$, saisie située dans l'époque passée (localisation temporelle dans laquelle le point de référence $\rho$ est antérieur à l'énonciation principale $S, \rho-S$ ).

Notre analyse permet de rendre compte de la défectivité de l'auxiliaire aller dans ce tour, à savoir du fait qu'il ne peut être conjugué qu'au présent et à l'imparfait, mais pas aux autres formes simples (passé simple, futur, conditionnel), ni à aucune des formes composées : 
- au présent et à l'imparfait, le point de référence $\rho$ est soit en relation sous-déterminée par rapport à l'intervalle $\varepsilon_{\mathrm{i}}-\varepsilon_{\mathrm{t}}$ (au présent $: \rho \subseteq \varepsilon_{\mathrm{i}}-\varepsilon_{\mathrm{t}}$ ), soit en relation d'inclusion dans l'intervalle $\varepsilon_{\mathrm{i}}-\varepsilon_{\mathrm{t}}$ (à l'imparfait $: \rho$ $\left.\subset \varepsilon_{\mathrm{i}}-\varepsilon_{\mathrm{t}}\right)$. Les relations de sous-détermination $\left(\rho \subseteq \varepsilon_{\mathrm{i}}-\varepsilon_{\mathrm{t}}\right)$ et d'inclusion $\left(\rho \subset \varepsilon_{\mathrm{i}}-\varepsilon_{\mathrm{t}}\right)$ peuvent parfaitement, dans les formes prospectives construites sur l'interaction de $\rho$ avec le V. à l'infinitif, se spécifier en relation d'antériorité immédiate par rapport à la borne finale $\left(\rho<\varepsilon_{t}\right)$ : du point de vue logique, l'antériorité immédiate est une spécification de la neutralité comme de l'inclusion; et comme $\varepsilon_{\mathrm{t}}$ coïncide avec $\mathrm{E}_{\mathrm{i}}$, on en déduit que $\rho<\mathrm{E}_{\mathrm{i}}$.

- L'impossibilité du passé simple : *Guillaume alla partir en vacances tient à ce que ce temps, du fait de la relation de coïncidence entre $\rho$ et l'intervalle $\varepsilon_{\mathrm{i}}-\varepsilon_{\mathrm{t}}$ réduit à un point $(\rho=\varepsilon)$, impliquerait, du fait de l'interaction de $\rho$ avec le $V$. à l'infinitif, la relation $\rho=E_{i}$ : le point de référence $\rho$ coïnciderait avec la borne initiale du procès $E$. Ne serait plus ménagée la relation d'antériorité proche $\left(\rho<\mathrm{E}_{\mathrm{i}}\right)$ nécessaire à la production du sens prospectif.

- L'absence de tours prospectifs au futur ( ?Guillaume ira partir en vacances) et au conditionnel ( ? Guillaume irait partir en vacances) tient non à une impossibilité aspectuelle mais à une difficulté temporelle : l'ultériorité serait marquée deux fois : par l'auxiliaire et par le temps verbal. Ce que le français se refuse de faire, mais pas le portugais du Brésil (Oliveira 2005) qui peut parfaitement actualiser, en sociolecte plutôt familier, l'énoncé il va pleuvoir au futur (irá chover) et au conditionnel (iria chover).

- L'impossibilité des temps composés (*Guillaume est allé partir en vacances) tient à ce que la forme

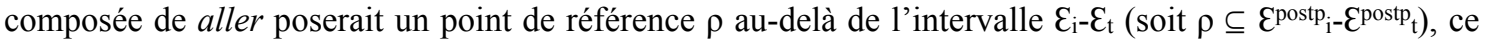
qui entraînerait que $\rho$ serait non pas proximalement antérieur à $E_{i}\left(\rho<E_{i}\right)$ comme demandé pour la production du sens de prospection, mais que $E_{i}$ serait proximalement antérieur à $\rho\left(E_{i}<\rho\right)$.

\subsubsection{Venir de + infinitif}

Venir (de), en tant que verbe, signifie, en appui sur la préposition de, le mouvement depuis un point donné (le train vient de Paris). Grammaticalisé en auxiliaire, il indique le mouvement depuis la phase processuelle du procès dénoté et permet de le saisir dans sa phase post-processuelle, à proximité de sa phase processuelle.

\begin{tabular}{|c|c|c|}
\hline Formes & Représentation aspectuelle & Localisation temporelle \\
\hline Infinitif & $\mathrm{R} \subseteq \mathrm{E}_{\mathrm{i}}-\mathrm{E}_{\mathrm{t}}$ & $\varnothing$ \\
\hline Venir de au présent & $\rho \subseteq \varepsilon_{\mathrm{i}}-\varepsilon_{\mathrm{t}}$ & $\rho=S$ \\
\hline$\rightarrow$ présent rétrospectif & $E_{t}<\rho$ & $\rho=S$ \\
\hline Infinitif & $\mathrm{R} \subseteq \mathrm{E}_{\mathrm{i}}-\mathrm{E}_{\mathrm{t}}$ & $\varnothing$ \\
\hline Venir de à l'imparfait & $\rho \subset \varepsilon_{\mathrm{i}}-\varepsilon_{\mathrm{t}}$ & $\rho-S$ \\
\hline$\rightarrow$ imparfait rétrospectif & $\mathrm{E}_{\mathrm{t}}<\rho$ & $\rho-S$ \\
\hline Infinitif & $\mathrm{R} \subseteq \mathrm{E}_{\mathrm{i}}-\mathrm{E}_{\mathrm{t}}$ & $\varnothing$ \\
\hline Venir de au futur & $\rho \subseteq \varepsilon_{\mathrm{i}}-\varepsilon_{\mathrm{t}}$ & $S-\rho$ \\
\hline$\rightarrow$ futur rétrospectif & $\mathrm{E}_{\mathrm{t}}<\rho$ & $S-\rho$ \\
\hline Infinitif & $\mathrm{R} \subseteq \mathrm{E}_{\mathrm{i}}-\mathrm{E}_{\mathrm{t}}$ & $\varnothing$ \\
\hline Venir de au conditionnel & $\rho \subseteq \varepsilon_{\mathrm{i}}-\varepsilon_{\mathrm{t}}$ & $S^{\prime}-S^{\prime} ; S^{\prime}-\rho$ \\
\hline$\rightarrow$ conditionnel rétrospectif & $\mathrm{E}_{\mathrm{t}}<\rho$ & $S^{\prime}-S^{\prime} ; S^{\prime}-\rho$ \\
\hline
\end{tabular}


Les formules données dans le tableau peuvent être explicitées ainsi :

Présent rétrospectif : Guillaume vient de partir en vacances : $E_{t}<\rho ; \rho=S$

Le présent rétrospectif, combinaison de l'auxiliaire venir + de au présent et du V. à l'infinitif, saisit le procès dans sa phase post-processuelle, en un point de référence $\rho$ à proximité de la borne terminale $E_{t}$ de phase processuelle du procès $\left(E_{t}<\rho\right)$, saisie située dans l'époque présente (localisation temporelle dans laquelle le point de référence $\rho$ coïncide avec l'énonciation principale $S, \rho=S$ ).

Imparfait rétrospectif : Guillaume venait de partir en vacances : $E_{t}<\rho ; \rho-S$

L'imparfait rétrospectif, combinaison de l'auxiliaire venir + de à l'imparfait et du V. à l'infinitif, saisit le procès dans sa phase post-processuelle, en un point de référence $\rho$ à proximité de la borne terminale $E_{t}$ de la phase processuelle du procès $\left(E_{t}<\rho\right)$, saisie située dans l'époque passée (localisation temporelle dans laquelle le point de référence $\rho$ est antérieur à l'énonciation principale $S, \rho-S)$.

A la différence de la forme périphrastique prospective, la forme périphrastique rétrospective dispose d'un futur et d'un conditionnel ${ }^{\text {xii }}$ ce qui s'explique dans la mesure où la difficulté temporelle qui interdisait les formes prospectives *ira / *irait partir n'est plus de mise ici puisque la forme périphrastique signifie la rétrospection :

Futur rétrospectif : Guillaume viendra de partir en vacances : $E_{t}<\rho ; S-\rho$

Le futur rétrospectif, combinaison de l'auxiliaire venir $+d e$ au futur et du V. à l'infinitif, saisit le procès dans sa phase post-processuelle, en un point de référence $\rho$ à proximité de la borne terminale $E_{t}$ de la phase processuelle du procès $\left(E_{t}<\rho\right)$, saisie située dans l'époque future (localisation temporelle dans laquelle l'énonciation principale est antérieure au point de référence, $S-\rho)$.

Conditionnel rétrospectif : Guillaume viendrait de partir en vacances : $E_{t}<\rho ; S^{\prime}-S ; S^{\prime}-\rho$

Le conditionnel rétrospectif, combinaison de l'auxiliaire venir + de au conditionnel et du V. à l'infinitif, saisit le procès dans sa phase post-processuelle, en un point de référence $\rho$ à proximité de la borne terminale $E_{t}$ de la phase processuelle du procès $\left(E_{t}<\rho\right)$. Cette saisie est située dans l'ultériorité d'une énonciation secondaire $\left(S^{\prime}-\rho\right)$, elle-même située dans l'antériorité de l'énonciation principale $\left(S^{\prime}-S\right)$. Notons que, comme dans le cas du conditionnel présent, le point de référence $\rho$ étant positionné par rapport à une énonciation $S$ ' antérieure à $S$, la relation entre $\rho$ et $S$ n'est pas contrainte.

Notre analyse permet de rendre compte de la défectivité de l'auxiliaire venir dans ce tour, à savoir du fait qu'il peut être conjugué aux présent, imparfait, futur, conditionnel présent mais ni au passé simple, ni à aucune des formes composées :

- aux présent, imparfait, futur, conditionnel présent, le point de référence $\rho$ est soit en relation sousdéterminée par rapport à l'intervalle $\varepsilon_{\mathrm{i}}-\varepsilon_{\mathrm{t}}$ (au présent, futur et conditionnel $: \rho \subseteq \varepsilon_{\mathrm{i}}-\varepsilon_{\mathrm{t}}$ ), soit en relation d'inclusion dans l'intervalle $\varepsilon_{\mathrm{i}}-\varepsilon_{\mathrm{t}}$ (à l'imparfait : $\rho \subset \mathcal{E}_{\mathrm{i}}-\varepsilon_{\mathrm{t}}$ ). Les relations de sous-détermination $\left(\rho \subseteq \varepsilon_{\mathrm{i}}-\varepsilon_{\mathrm{t}}\right)$ et d'inclusion ( $\left.\rho \subset \varepsilon_{i}-\varepsilon_{t}\right)$ peuvent parfaitement, dans les formes rétrospectives construites sur l'interaction de $\rho$ avec le V. à l'infinitif, se spécifier en relation d'antériorité immédiate de la borne initiale par rapport à $\rho\left(\varepsilon_{\mathrm{i}}<\rho\right):$ du point de vue logique, l'antériorité immédiate est une spécification de la neutralité comme de l'inclusion; et comme $\varepsilon_{\mathrm{i}}$ coïncide avec $\mathrm{E}_{\mathrm{t}}$, on en déduit que $\mathrm{E}_{\mathrm{t}}<\rho$.

- L'impossibilité du passé simple : *Guillaume vint de partir en vacances tient à ce que ce temps, du fait de la relation de coïncidence entre $\rho$ et l'intervalle $\varepsilon_{\mathrm{i}}-\varepsilon_{\mathrm{t}}$ réduit à un point $(\rho=\varepsilon)$, impliquerait, du fait de l'interaction de $\rho$ avec le $\mathrm{V}$. à l'infinitif, la relation $\rho=\mathrm{E}_{\mathrm{t}}$ : le point de référence $\rho$ coïnciderait avec la borne terminale $E_{t} d u$ procès $E$. Ne serait plus ménagée la relation d'antériorité proche $\left(E_{t}<\rho\right)$ nécessaire à la production du sens rétrospectif. 
- L'impossibilité des temps composés (*Guillaume est venu de partir en vacances) tient à ce que la forme composée de venir poserait un point de référence $\rho$ au-delà de l'intervalle $\varepsilon_{\mathrm{i}}-\varepsilon_{\mathrm{t}}$ (soit $\rho \subseteq \mathcal{E}^{\text {postp }_{\mathrm{i}}-}$

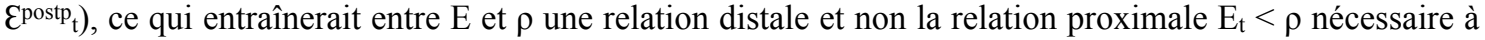
la production du sens de rétrospection.

\section{Phases et formes}

Nous avons dit que la phase processuelle était signifiée par les formes simples ; les phases pré- et postprocessuelles, par les formes composées et périphrastiques. En français, ce couplage est subverti en deux points de recomposition diachronique du système : le passé composé ; le présent et l'imparfait prospectifs :

- le passé composé, qui en tant que forme composée prend en charge la phase post-processuelle (valeur d'accompli du présent, selon les grammaires), a concurrencé au fil des siècles la forme simple du passé simple, pour prendre également en charge la phase processuelle (valeur d'aoriste du discours (Benveniste 1966 [1959]);

- le présent et l'imparfait prospectifs, qui en tant que formes périphrastiques prennent en charge la phase pré-processuelle, concurrencent les formes simples (futur pour le présent prospectif ; conditionnel pour l'imparfait prospectif) pour prendre également en charge la phase processuelle.

Le français fait du temps avec de l'aspect : le passé composé fonctionne comme un temps de l'époque passée ; le présent prospectif comme un temps de l'époque future ; et use de certaines formes composées ou périphrastiques pour signifier la phase processuelle. Ce fait est commun dans nombre de langues, notamment dans les langues romanes. Pour ne prendre qu'un exemple : le catalan a vu la forme synthétique du prétérit concurrencée par une forme périphrastique construite sur aller au présent : «El Guillem va marxar de vacances » (littéralement : 'Guillaume va partir en vacances'), un tour que le français a connu jusqu'au XVI ème siècle et que l'occitan a conservé dans certains dialectes.

\section{Conclusion}

Notre propos était de tenter de décrire, à partir d'un nombre fini de paramètres, le système des temps de l'indicatif, en retravaillant la modélisation de Reichenbach (1947).

Selon la modélisation que nous venons de présenter, les temps verbaux sont analysables à partir des trois éléments $\mathrm{E}, \mathrm{S}$ et $\mathrm{R}$ et de leurs relations redéfinis comme suit :

(i) $\mathrm{E}$, correspondant au procès, est un intervalle borné $\left(\mathrm{E}_{\mathrm{i}}-\mathrm{E}_{\mathrm{t}}\right)$ (pouvant éventuellement se réduire à un point, comme dans les cas du passé simple). Le procès peut être représenté dans sa phase processuelle ( $\mathrm{E}_{\mathrm{i}}$ -

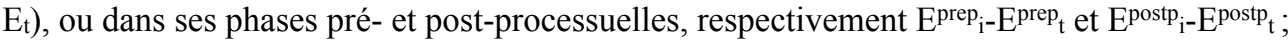

(ii) $\mathrm{S}$ et $\mathrm{R}$ sont des points correspondant pour le premier au to de l'énonciation et pour le second au point de référence à partir duquel est représenté le procès ;

(iii) pour les formes simples, la représentation aspectuelle du procès procède de la relation entre $\mathrm{R}$ et $\mathrm{E}$; la localisation temporelle, de la relation entre $\mathrm{R}$ et $\mathrm{S}$;

(iv) les relations entre $\mathrm{R}$ et $\mathrm{E}$ peuvent être d'antériorité $(-)$, de coïncidence $(=)$, d'inclusion $(\subset)$, de neutralité $(\subseteq)$ et d'antériorité proche $(<)$; les relations entre $\mathrm{R}$ et $\mathrm{S}$ peuvent être d'antériorité $(-)$ ou de coïncidence $(=)$;

(v) le fonctionnement dialogique du conditionnel demande de poser, complémentairement à $S$, un second point d'énonciation, $S^{\prime}$. Ce second point est localisé temporellement par rapport à $S$ ( $S$ '-S), ce qui introduit un élément d'hétérogénéité dans le système des relations posé en (iv). Cet élément d'hétérogénéité pointe peut-être là le talon d'Achille de notre modélisation ; 
(vi) la phase processuelle $\left(\mathrm{E}_{\mathrm{i}}-\mathrm{E}_{\mathrm{t}}\right)$ est prototypiquement représentée par les formes simples ; la phase pré-

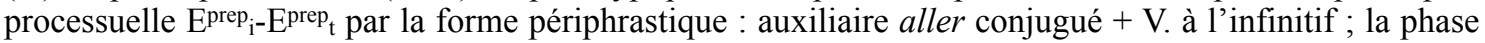

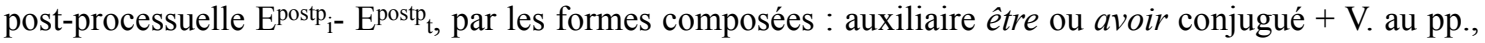
et par la forme périphrastique : auxiliaire venir conjugué $+d e+\mathrm{V}$. à l'infinitif ;

(vii) les auxiliaires aller, être, avoir, venir sont dotés d'un intervalle de procès : $\varepsilon_{\mathrm{i}}-\varepsilon_{\mathrm{t}}$. Cet intervalle coïncide pour aller avec l'intervalle pré-processuel $\mathrm{E}^{\text {prep }_{\mathrm{i}}-\mathrm{E}^{\mathrm{prep}} \mathrm{t}} \mathrm{du} \mathrm{V}$; ; et pour être, avoir, venir, avec

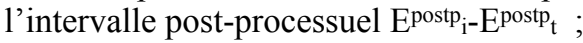

(viii) l'intervalle $\varepsilon_{\mathrm{i}}-\varepsilon_{\mathrm{t}}$ de l'auxiliaire est doté d'un point de référence $\rho$;

(ix) pour les formes composées et périphrastiques, la représentation aspectuelle s'effectue par l'interaction de la relation entre $\mathrm{E}$ et $\mathrm{R}$ d'une part, et entre $\varepsilon \mathrm{E}-\varepsilon \mathrm{E}$ et $\rho$ d'autre part ; interaction qui a pour

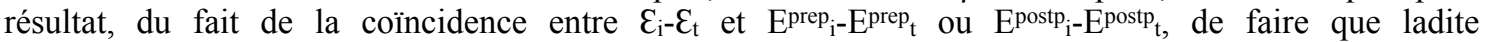

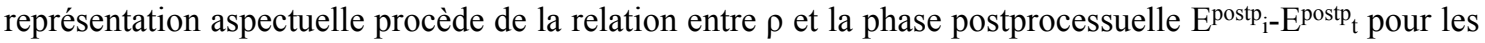
formes composées ; de la relation entre $\rho$ et la phase processuelle E pour les formes périphrastiques ;

(x) pour les formes composées et périphrastiques, étant donné que le V. à l'infinitif ou au pp. ne marque pas le temps, la localisation temporelle procède de la relation entre $\rho$ et $S$.

Ce système pourra paraître bien complexe, et nous ne le nierons pas. Complexification inutile du système de Reichenbach ? Nous ne le croyons pas. La complexité procède de l'élément que nous considérons comme essentiel de notre analyse : l'attribution à l'auxiliaire d'un intervalle de procès $\varepsilon_{\mathrm{i}}-\varepsilon_{\mathrm{t}}$ correspondant

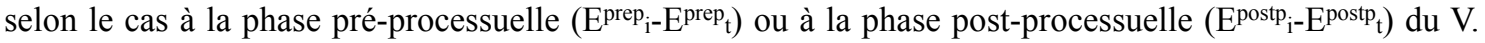
Et conséquemment, l'attribution à cet intervalle de procès d'un point de référence, $\rho$. C'est la relation

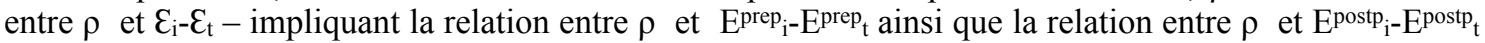
- qui seule permet de rendre compte :

- de la différence aspectuelle passé antérieur $\left(\rho=E^{\text {postp }}\right) /$ plus-que-parfait $\left(\rho \subset E^{\text {postp }_{i}-E^{\text {postp }}}{ }_{t}\right)$;

- de la défectivité de aller/venir en tant qu'auxiliaires temporels.

La modélisation proposée est celle de la valeur en langue des temps de l'indicatif. Sa robustesse se vérifiera de sa capacité à rendre compte de l'interaction de l'aspect grammatical avec l'aspect lexical des verbes (p. ex. pourquoi venir de + inf. ne peut guère actualiser un état permanent : ?elle venait d'avoir les yeux verts) ; ainsi que des différents emplois en discours que peuvent prendre tel ou tel temps, p. ex. des effets de sens particuliers comme l'effet de sens narratif de l'imparfait (Le lendemain Guillaume partait en vacances) ou comme l'effet de sens conjectural du futur (Guillaume n'est pas chez lui, il sera parti en vacances). Les premières recherches effectuées dans ce sens, que nous ne pouvons présenter ici, semblent valider la modélisation.

\section{Références bibliographiques}

Beauzée, N. (1782). Temps. Encyclopédie méthodique : grammaire et littérature, reproduit dans Swiggers P., 1986, Grammaire et théorie du langage au XVIII'me siècle : " mot", "temps ", " mode dans l'Encyclopédie méthodique. Lille : Presses universitaires de Lille, 67-81.

Benveniste, É. (1966 [1959]). Les relations de temps dans le verbe français. Problèmes de linguistique générale. Paris: Gallimard. 237-257.

Bres, J. (2009). Dialogisme et temps verbaux de l'indicatif. Langue Française, 163, 21-39.

Bres, J. (2015). De la défectivité de aller et de venir dans les périphrases d'ultériorité (il va pleuvoir) et d'antériorité (il vient de pleuvoir) proches à l'indicatif. L'Information grammaticale,144, 27-33.

Bres J. et Labeau E., 2013, «Aller et venir : des verbes de déplacement aux auxiliaires aspectuels-temporelsmodaux », Langue française 179, 13-28.

Comrie, B. (1976). Aspect. Cambridge : Cambridge University Press.

Comrie, B. (1981). On Reichenbach's approach to tense. CLS, 17, 24-30. 
Comrie, B. (1985). Tense. Cambridge : Cambridge University Press.

Damourette, J. et Pichon, E. (1970[1911-1936]). Des mots à la pensée : essai de grammaire de la langue française. Paris : d'Artrey.

Declerck, R. (1986). From Reichenbach (1947) to Comrie (1985) and beyond. Towards a Theory of Tense. Lingua, 70, 305-364.

Gosselin, L. (2005). Temporalité et modalité. Bruxelles : De Boeck Duculot.

Gosselin, L. (1996). Sémantique de la temporalité en français : un modèle calculatoire et cognitif du temps et de l'aspect. Louvain-la-Neuve : Duculot.

Guillaume, G. (1929[1970]). Temps et verbe. Paris : Champion.

Guillaume, G. (1964[1933]. Immanence et transcendance dans la catégorie du verbe. Langage et sciences du langage. Paris :Nizet, 46-58.

Klein, W. (1994). Time in language. London : Routledge.

Martin, R. (1971). Temps et aspect. Essai sur l'emploi des temps narratifs en moyen français. Paris : Klinsieck.

Reichenbach, H. (1947). Elements of Symbolic Logic. New York : Macmillan \& Co.

Saussure de, L. (2010). Pragmatique procédurale des temps verbaux : la question des usages interprétatifs. Flaux, N., Stosic, D. \& Vet, C. (eds.). Interpréter les temps verbaux. Peter Lang, 129-160.

Saussure de, L. (1998). L'approche référentielle: de Beauzée à Reichenbach. Moeschler, J. \& al. (dir). Le temps des événements. Pragmatique de la référence temporelle. Paris : Kimé, 19-44.

Vet, C. (1980). Temps, aspects et adverbes de temps en français contemporain. Essai de sémantique formelle. Genève : Droz.

Vet, C. (2007). The descriptive inadequacy of Reichenbach's tense system : A new proposal. Cahiers Chronos 17: $7-26$.

Vetters, C. (1996). Temps, aspect et narration. Amsterdam/Atlanta : Rodopi.

Vetters, C. (2002). Remarques sur l'analyse reichenbachienne des temps verbaux. Romanica Wratislaviensia, 49, 93-132.

Vikner, S. (1985). Reichenbach revisited : one, two, or three temporal relations ?. Acta Linguistica Hafniensia, 19.2, 81-98.

Wilmet, M. (1970). Le système de l'indicatif en moyen français. Genève : Droz.

Un premier temps de cette recherche s'est fait en interaction avec L. Gosselin. Qu'il en soit ici remercié.

ii Reichenbach ne donne pas de nom à cette indication complémentaire qu'il représente par un petit tertre dans le schéma 1.

iii On emploiera le terme de formes (simples, composées, périphrastiques) lorsqu'on traitera de la morphologie et le terme de temps pour désigner les tiroirs : présent, passé composé, imparfait, etc.

iv Ce qui n'était pas le cas du latin, qui signifiait la phase post-processuelle par une forme synthétique construite sur le perfectum : amavero, j'aurai aimé.

v Nous ne prendrons en compte ici que ces deux auxiliaires + inf. Au cours de son histoire, la langue a développé de nombreuses formes, qui ont été ensuite parfois abandonnées. Cf. Gougenheim 1929. Notons que l'auxiliaire être en train de (+ V. inf.), actuellement bien vivant, permet de saisir la phase processuelle. Il en allait de même pour aller + gérondif (" la fauve passion va sonnant l'olifant ! ", P. Verlaine, Poèmes saturniens, 1896), cf. Bres et Labeau 2013). Un prochain travail traitera, dans le cadre analytique proposé ici, des différents tours périphrastiques. 
vi Nous analysons les formes composées comme des formes périphrastiques qui sont plus avancées sur le chemin de grammaticalisation du verbe en auxiliaire.

Il nous semble significatif que les critiques (notamment Vetters 2002) du système aient porté sur ce temps.

Nous parlons de temps dialogique en langue en ce que le conditionnel, du fait qu'il est issu de la grammaticalisation d'une périphrase composée de l'infinitif et d'un auxiliaire à l'imparfait, fait intervenir deux énonciations : il situe le procès en ultériorité par rapport à une énonciation secondaire, elle-même située dans l'antériorité de l'énonciation principale.

ix On parle d'emploi dialogique pour des occurrences comme :

Mais n'est-il pas plus simple que j'aille à Paris ? Ma mère pourra trouver un prétexte pour m'y envoyer : ce sera un oncle qui me demande, une tante en train de mourir. (H. de Balzac, La Vieille fille, 1837)

Ce qui est posé comme ultérieur au point d'énonciation $S$, ce n'est pas le point de référence $R$ du procès « sera » $(\mathrm{S}-\mathrm{R})$, mais une énonciation ultérieure $\mathrm{S}$ 'implicite $(\mathrm{S}-\mathrm{S}$ '). L'énoncé équivaut à : elle dira que c'est un oncle qui me demande.

x Pour plus de lisibilité, nous représenterons la relation de coïncidence par le signe $=$. Il nous paraît un symbole plus parlant que le signe (,) employé par Reichenbach, qui prête facilement à confusion en ce sens qu'il peut signifier une succession.

xi Nous expliquons infra 2.2.2. pourquoi l'intervalle E est dans ce cas réduit à un point.

xii Pour répondre à la question d'un évaluateur : venir de s'utilise sans forçage aucun au futur comme au conditionnel :

(a) Alors il fera ce qu'il voudra, naturellement, et je serai, comme elle le veut « bien gentil ». Mais vous avouerez que pour un type qui viendra de passer cinq mois sous l'autorité militaire ça n'est pas une atmosphère très marrante. (J. - P. Sartre, Lettres au Castor, 1940-1963)

(b) Il y a une heure dans toute vie où l'on rencontre, désarmé, sa vérité nue telle une baïonnette qui viendrait de jaillir du fourreau. (P. Mertens, Les Eblouissements, 1987) 